\title{
NBSIR 79-1614
}

\section{EVALUATION OF SIGNAL PLUS NOISE DETECTION ERROR IN AN ENVELOPE DETECTOR WITH LOGARITHMIC COMPRESSION}

\author{
A. J. Estin \\ W. C. Daywitt
}

Electromagnetic Fields Division National Engineering Laboratory National Bureau of Standards Boulder, Colorado 80303

August 1979

100

.456

79-1u14 



\title{
NBSIR 79-1614 \\ ...
}

\section{EVALUATION OF SIGNAL PLUS NOISE DETECTION ERROR IN AN ENVELOPE DETECTOR WITH LOGARITHMIC COMPRESSION}

\author{
A. J. Estin \\ W. C. Daywitt
}

Electromagnetic Fields Division

National Engineering Laboratory

National Bureau of Standards

Boulder, Colorado 80303

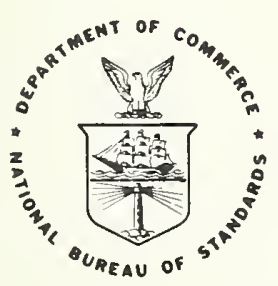

U.S. DEPARTMENT OF COMMERCE, Juanita M. Kreps, Secretary Luther H. Hodges, Jr., Under Secretary Jordan J. Baruch, Assistant Secretary for Science and Technology 

1. INTRODUCTION

1.1 Background

1.2 Review of Random Processes

1.3 The Envelope Function

2. FORMULATION OF THE PROBLEM

2.1 The Basic PDF

2.2 Analysis

3. EXPERIMENTAL RESULTS

4. USING THE CORRECTION FACTOR

5. ACKNOWLEDGMENT AND NOTE

6. REFERENCES

6.1 General Reference 



\section{A. J. Estin and W. C. Daywitt}

A correction factor is derived for the detected output of a modulated sinusoidal signal with added Gaussian noise, as processed by an envelope detector with logarithmic compression which is calibrated for a sinusoid. Supporting experimental data are presented which were obtained using a typical commercial system having such a detector.

Key words: Detection; detection amplitude error; noisy signal detection.

\section{INTRODUCTION \\ 1.1 Background}

Signal detection techniques in various measurement systems serve to convert a signal into a relatively slowly varying voltage which is intelligible and useful. One price paid in this process is that no one signal detection technique will so convert all different signal waveforms without introducing some intrinsic amount of error, possibly major. For example, a root-mean-square detector will accurately represent power of the signal; but if peak amplitude of a short rectangular pulse of low duty cycle is desired, a correction factor must be applied to remove the error, this factor being a function of both the signal characteristics and the detector properties.

One very common and useful detection system is that which envelope detects a received signal and then logarithmically compresses the result. Such a system is fast, stable, economical, sensitive, has wide frequency and dynamic ranges, and provides a convenient output expressed in decibels. It is usually calibrated for a sinusoidal waveform and is used in a large number of commercially available spectrum analyzers and frequency-selective voltmeters.

In this report, we will analyze the detection error in this type of detector for the case of a modulated sinusoid superimposed on Gaussian noise:

\subsection{Review of Random Processes}

Signals representing time-varying physical phenomena are usually classified as "deterministic" when they can be described by an explicit mathematical function of time, and "nondeterministic" or "random" when such an exact description is not possible. Even in the latter case, however, certain characterizations are possible based upon the facts that a range of values that the variable can assume may be known, and the probability of it having each value of this range may be determined. If we are fortunate enough to

* Work partially supported by the U.S. Army Satellite Communications Agency. 
have this information, many useful predictions can be made about such random variables. A function describing this probability distribution for a continuous random variable ("continuous" in range, not in sequence of values) is the "Probability Density Function," or PDF, which we designate as $P_{\xi}(x)$. $P_{\xi}(x) d x$ is a real non-negative function whose value is the relative probability of the event that $\xi$ will lie in the interval $(x, x+d x)$. It follows that the probability that $\xi$ falls in the interval $\left(x_{1}, x_{2}\right)$ is

$$
\int_{x_{1}}^{x_{2}} P_{\xi}(x) d x
$$

with the total probability being normalized to unity. The PDF can be related to various observable quantities by means of integrals with appropriate weighting functions over its range. If the weighting function is chosen as $x^{n}$, the observable is the nth moment of the PDF:

$$
\left\langle\xi^{n}\right\rangle=\int_{-\infty}^{\infty} x^{n} P_{\xi}(x) d x
$$

where the angular bracket denotes the expected value of the argument.

In particular, the first moment represents its average value and if $\xi$ is a voltage across a unit resistance, the second moment represents the power dissipated in the resistor. The variance of $\xi$ is

$$
\sigma_{\xi}^{2}=\left\langle\xi^{2}\right\rangle-\langle\xi\rangle^{2}
$$

and $\sigma_{\xi}$ is the standard deviation, which is a measure of the dispersion about the mean.

\subsection{The Envelope Function}

The concept of the envelope of a voltage waveform is intuitively understood to be that function which connects the successive positive (or negative) peaks of, for example, a sinusoid that varies slowly in amplitude and/or phase. For any but the simplest waveforms, precise application of this definition encounters considerable difficulties. If the Fourier transform of the time series representing the waveform contains significant components far above and below the midband frequency, the envelope definition becomes subject to the exact choice of mid-frequency. If several uncorrelated time series were combined, the non-linear RMS process becomes difficult to 
calculate, and in particular if these time series were random, the result would rapidly become uncalculable.

A rigorous generalization of the envelope function has been obtained in terms of Hilbert transforms [1]. If $h(t)$ is a real time series, its corresponding pre-envelope (or pre-detection) function $z(t)$ is given by

$$
z(t)=h(t)+j \hat{h}(t)
$$

where $\hat{h}(t)$ is the Hilbert transform of $h(t)$. The envelope is defined as the absolute value of $z(t)$ :

$$
|z(t)|=\sqrt{z(t) z^{*}(t)}
$$

where the asterisk denotes the complex conjugate. It will be useful to show, at this time, that the power in a time series which is Fourier-expandable is related to the envelope function by:

$$
P(t)=\frac{|z(t)|^{2}}{2} \text {. }
$$

If $s(t)$ is a voltage time series, which can be expressed as a Fourier series, then

$$
s(t)=\sum_{n} a_{n} \cos \omega_{n} t+b_{n} \sin \omega_{n} t \text {. }
$$

In order to obtain a power that is "instantaneous" with respect to the envelope variations, we shall integrate over a time $\Delta T$ that is long compared with any $r f$ period $2 \pi / \omega_{n}$, but short compared with any modulation period $2 \pi /\left(\omega_{n}-\omega_{m}\right)$. (This constrains us to the case when the signal bandwidth is small relative to its rf components.) 
Hence,

or

$$
P(T)=\frac{1}{\Delta T} \int_{T}^{T+\Delta T} s^{2}(t) d t
$$

$$
\begin{aligned}
P(T)=\frac{1}{2} \sum_{n} \sum_{m} & \left\{\left(a_{n m} a_{n}+b_{n m} b_{m}\right) \cos \left(\omega_{n}-\omega_{m}\right) T+\right. \\
& \left.\left(a_{m} b_{n}-a_{n m} b_{m}\right) \sin \left(\omega_{n}-\omega_{m}\right) T\right\}
\end{aligned}
$$

The Hilbert transforms of the sine and cosine functions are: [2]

$$
\left.\begin{array}{ll}
g_{1}(t)=\sin \omega t, & \hat{g}_{1}(t)=\cos \omega t \\
g_{2}(t)=\cos \omega t, & \hat{g}_{2}(t)=-\sin \omega t
\end{array}\right\}
$$

From eqs (3), (6), and (8),

$$
z(t)=\sum_{n}\left(a_{n}+j b_{n}\right) e^{-j \omega_{n} t}
$$

It follows that $\left|z(t)^{2}\right| / 2$ is equal to the right side of eq (7).

\section{FORMULATION OF THE PROBLEM}

\subsection{The Basic PDF}

The PDF for the envelope of Gaussian noise added to a signal composed of a sum of sinusoids is given by the Rice-Nakagami relation [3],

$$
\begin{aligned}
P_{\xi}(x) & =\frac{x}{\psi_{0}} I_{0}\left(\frac{x|z(t)|}{\psi_{0}}\right) \exp \left(\frac{x^{2}+|z(t)|^{2}}{-2 \psi_{0}}\right), & x \geq 0 \\
& =0 & , x<0
\end{aligned}
$$


where: $z(t)$ is the signal function, $\psi_{0}$ is the mean-squared value of the Gaussian noise power, and $I_{0}$ is the modified Bessel function of the first kind and order zero.

Note that $|z(t)|$ is the envelope function of the signal in the absence of noise. (For convenience, we are henceforth dropping the subscripts used in eq (1) and replacing $z(t)$ by $z_{\text {.) }}$ It is helpful to examine the limiting cases of eq (9) for high and low signal-to-noise $(S / N)$ power ratios.

Since $I_{0}(0)=1$, the low $S / N$ imit is:

$$
\lim _{z \rightarrow 0} P(x)=\frac{x}{\psi_{0}} \exp \left(-\frac{x^{2}}{2 \psi_{0}}\right)
$$

This is the familiar Rayleigh distribution for band-limited noise.

Using the asymptotic form of $I_{0}(u)$ for very large argument,

$$
I_{0}(u) \sim \frac{e^{u}}{\sqrt{2 \pi u}}
$$

and with the definition $q \equiv 1 /\left(2 \psi_{0}\right)$, the limit for high $S / N$ ratio is:

$$
\lim _{q+\infty} P(x)=\lim _{q \rightarrow \infty} \sqrt{\frac{q x}{\pi|z|}} \exp \left\{-q(x-|z|)^{2}\right\} .
$$

This is recognizable as the Dirac delta function [4]. Hence,

$$
\lim _{\substack{\psi \rightarrow 0 \\ 0}} P(x)=\sqrt{\frac{x}{|z|}} \delta(x-|z|)
$$

and as might be anticipated, in the absence of noise, the probability distribution is concentrated at the signal envelope. 


\subsection{Analysis}

We will next develop a rigorous expression for the signal-to-noise power ratio. The white noise power delivered into the detector through a filter of bandpass characteristic $H(f)$ is

$$
\psi_{0}=\frac{1}{2} \int_{-\infty}^{\infty}|H(f)|^{2} W_{0} d f=W_{0} B
$$

where $W_{0}$ is the noise power density (watts/Hertz) and $B$ is the equivalent noise bandwidth.

If the modulated carrier is assumed to be of the form of eq (6), in which the sidebands lie within a constant gain portion of $H(f)$, the power in the carrier is given by eq (5). Thus, the signal-to-noise power ratio is

$$
r=\frac{|z|^{2}}{2 \psi_{0}}
$$

Letting $x$ represent the random variable of the detected signal, i.e., the envelope variable, then the logarithmically compressed output is given by

$$
y=\ln x
$$

The average value of this output is its first moment, which from eq (1) is

$$
\langle y\rangle=\int_{-\infty}^{\infty} y P(y) d y
$$

Since $y$ has a one-to-one correspondence with $x$, as shown in eq (12), its PDF can be obtained [5] from the PDF of $x$ as

$$
P(y) d y=P(x) d x
$$

Substituting eqs (12) and (14) into eq (13), and reducing the limits to correspond to the range of $y$, 


$$
\langle y\rangle=\int_{0}^{\infty} \ln x P(x) d x
$$

Combining eqs (9) and (11) into eq (15), and defining a new variable of integration $w \equiv x^{2} /\left(2 \psi_{0}\right)$, we have an expression for the average value of the output of the detected, logarithmically compressed voltage:

$$
\langle y\rangle=\frac{e^{-r}}{2}\left\{\ln \left(2 \psi_{0}\right) \int_{0}^{\infty} I_{0}(2 \sqrt{w r}) e^{-w} d w+\int_{0}^{\infty} \ln w I_{0}(\sqrt[2]{w r}) e^{-w} d w\right\} .
$$

In order to eliminate $\psi_{0}$ from this expression, we shall evaluate the power before logarithmic compression. Again, using eq (1),

$$
\left\langle x^{2}\right\rangle=\int_{0}^{\infty} \frac{x^{3}}{\psi_{0}} I_{0}\left(\frac{x|z|}{\psi_{0}}\right) \exp \left(\frac{x^{2}+|z|^{2}}{-2 \psi_{0}}\right) d x
$$

This can be integrated directly in terms of the, confluent hypergeometric function [6] as

$$
\left\langle x^{2}\right\rangle=2 \psi_{0}{ }_{1} F_{1}\left(-1 ; 1 ;-|z|^{2} / 2 \psi_{0}\right),
$$

which reduces to a finite two-term series:

$$
\left\langle x^{2}\right\rangle=2\left(\psi_{0}+\frac{|z|^{2}}{2}\right)
$$

Alternatively, eq (18) could have been obtained directly by appealing to the following theorem [7]: "The ensemble average of the square of the envelope function is equal to twice the ensemble average of the square of the original time series."

Solving eq (18) for $2 \psi_{0}$, and with eq (11),

$$
2 \psi_{0}=\frac{\left\langle x^{2}\right\rangle}{1+r}
$$


Substituting eq (19) into eq (16), and solving for the power in the detected envelope,

$$
\left\langle x^{2}\right\rangle=\exp \left\{\frac{2\langle y\rangle-e^{-r} I_{2}}{e^{-r} I_{1}}+1 n(1+r)\right\}
$$

where $I_{1}$ and $I_{2}$ are the following integrals:

$$
\begin{aligned}
& I_{1}=\int_{0}^{\infty} e^{-w} I_{0}(2 w r) d w \\
& I_{2}=\int_{0}^{\infty} e^{-w} \ln w I_{0}(2 w r) d w .
\end{aligned}
$$

The first integral can be easily evaluated in closed form. First, we expand the Bessel function in its power series (Dwight 813.1 )* as

$$
I_{0}(2 \sqrt{w r})=\sum_{n=0}^{\infty} \frac{(w r)^{n}}{(n !)^{2}} \text {. }
$$

Substituting this into eq (21), interchanging the order of summation and integration, and removing the quantities independent of the integration, we find that the integral reduces to the gamma function, $\Gamma(n+1)$, which, for integral $n$ becomes $n !$. The remaining series is identified as the exponential $e^{r}$, giving

$$
e^{-r_{1}}=1
$$

The second integral, eq (22), is somewhat more cumbersome. Again, we substitute eq (23) for the Bessel function and interchange the order of

* Dwight, H. B., Tables of Integrals and Other Mathematical Data (MacMillan, N.Y., 1957). 
integration and summation, obtaining

$$
I_{2}=\sum_{n=0}^{\infty} \frac{r^{n}}{\left(n_{0}^{\prime}\right)^{2}} \int_{0}^{\infty} e^{-w^{n}} \ln w d w .
$$

We designate this integral as $I_{2}(n)$; using $u=e^{-W}$ and $d v=w^{n}$ In $w d w$, this can be integrated by parts (Dwight 610.9) to produce a recursion relation in $n$.

$$
I_{2}(n+i)=(n+1) I_{2}(n)+n !, n \geq 0
$$

Since $I_{2}(0)=\gamma($ Dwight 852.1), where $\gamma$ is Euler's Constant = $0.5772156649 \ldots, I_{2}(n)$ can be obtained explicitly as

$$
I_{2}(n)=n ![-\gamma+H(n)]
$$

where $H(n)$ is the harmonic function

$$
H(n)=1+1 / 2+\ldots+1 / n \text {, and } H(0)=0 \text {. }
$$

Substituting eq (26) into eq (25), the factorial cancels one of those in the denominator, and we have for $\mathrm{I}_{2}$ :

$$
I_{2}=-\gamma e^{r}+\sum_{n=0}^{\infty} \frac{r^{n}}{n !} H(n), \quad H(0)=0 .
$$

Substituting eqs (24) and (27) into eq (20), and taking the natural logarithm of both sides, we finally arrive at the following expression:

$$
1 n\left\langle x^{2}\right\rangle=2\langle y\rangle+[\gamma+1 n(1+r)-\zeta(r)]
$$

where

$$
\zeta(r)=e^{-r} \sum_{n=1}^{\infty} \frac{r^{n}}{n !} H(n) .
$$

Each term of eq (28) is in nepers. The left-hand side represents the true power in the detected waveform. The first term on the right-hand side is the power indicated by the detector, and the term in square brackets is the 
desired correction term which depends only upon the signal-to-noise power ratio.

Unfortunately, the series in eq (29a) is slow and cumbersome to evaluate. We can, however, convert it to a much more tractable form in the following way. Let us differentiate $\zeta(r)$ with respect to $r$ (interchanging the order of summation and differentiation), and collect terms. Again, identifying the power series for the exponential, this has a simple form:

$$
\frac{d \zeta(r)}{d r}=\frac{1-e^{-r}}{r}
$$

To regain $\zeta(r)$, we integrate from a to $r$, with a to be determined as a constant of integration:

$$
\zeta(r)=\int_{a}^{r} \frac{1-e^{-r}}{r} d r .
$$

In order that $\zeta(0)=0$, as can be seen from eq (29a), it follows that $a \rightarrow 0$. By first expanding $e^{-r}$ in a power series, then integrating term-by-term, and finally letting $a \rightarrow 0$, we arrive at a much more convenient form for $\zeta(r)$ :

$$
\zeta(r)=\sum_{n=1}^{\infty} \frac{(-1)^{n+1} r^{n}}{n n !}
$$

This series is absolutely and uniformiy convergent for finite $r$, and furthermore may be truncated with any desired maximum error because it is alternating. Computation for very large $r$, however, may still prove difficult. The magnitude of the terms of the series will increase from $r$ (for the first term) to the order of $e^{r_{r}-3 / 2} / \sqrt{2 \pi}$ (for the $(r-2)^{\text {th }}$ or $(r-1)^{\text {th }}$ term) and only thereafter converge. Inasmuch as the computation process requires subtraction of successive terms, this could demand retention of an enormous number of significant figures for only modest accuracy in the result. To circumvent this difficulty, we return to eqs (29b) and (28). The correction term in eq (28), which we designate

$$
F(r)=\gamma+\ln (1+r)-\zeta(r)
$$




$$
F(r)=\ln \left(\frac{1+r}{r}\right)-E_{1}(r)
$$

where $E_{1}(r)$ is the real limit of the exponential integral (A\&S 5.1.11)*. An asymptotic expansion of eq (31) for large $r$ is easily obtained (A\&S 5.1.51, Dwight 601) as

$$
F(r)=r^{-1}-r^{-2}+\ldots+\frac{e^{-r}}{r}\left[1-r^{-1}+r^{-2}-\ldots\right]
$$

or

$$
F(r) \sim \frac{1}{r}, \quad r \gg>1
$$

For small $r$, we use the series expansion of $\ln (1+r)$. Combining with the terms of eq (29c) into eq (30):

$$
F(r)=\gamma-\frac{r^{2}}{4}+\frac{5 r^{3}}{18}-\ldots, \quad r \ll 1
$$

Values of $F(r)$ over the midrange of $r$ are readily evaluated from the exact solution

$$
F(r)=\gamma+\ln (1+r)-\sum_{n=1}^{\infty} \frac{(-1)^{n+1} r^{n}}{n n !}
$$

For convenience, these expressions for $F(r)$ are collected following:

* Abramowitz, M., and Stegun, I. A., Handbook of Mathematical Functions (NBS Applied Math Series No. 55, 1964). 


$$
\begin{aligned}
& F(r) \sim \frac{1}{r}, \quad r \gg 1 \\
& F(r)=\gamma-\frac{r^{2}}{4}+\frac{5 r^{2}}{18}-\ldots, \quad r \ll 1 \\
& F(r)=\gamma+\ln (1+r)-\sum_{n=1}^{\infty} \frac{(-1)^{n+1} r^{n}}{n n^{\prime} \cdot}, \quad r<\infty
\end{aligned}
$$

where $\gamma=$ Euler's Constant $=0.5772156649 \ldots$, and $F(r)$ is in nepers. To express this correction in decibels,

$$
F_{d B}(r)=\left(\operatorname{lol}_{10} e\right) F(r)
$$

and as a multiplicative ratio correction,

$$
\varepsilon(r)=e^{F(r)} \text {. }
$$

Alternative computational forms are available using polynomial expansions for the exponential integral form of eq (31). (A\&S 5.1.53-5.1.56)

\section{EXPERIMENTAL RESULTS}

The commercial automatic spectrum analyzers are typical instruments using the detection system analyzed in this paper. Response data were obtained with one of the most advanced such instruments, using as input a variable noise source (solid state diode followed by a calibrated attenuator) and a nominally calibrated signal generator. These data are shown in figure 1 . The solid line calculated curve was obtained from eq (32a) for $r>10$, eq (32b) for $r<0.1$, and eq $(32 \mathrm{c})$ for the intermediate range $0.1<r<10$.

\section{USING THE CORRECTION FACTOR}

It is apparent that making the correction described in this paper presupposes a knowledge of the signal and noise levels being measured. Most frequently this is not the case; and, in fact, finding the signal-to-noise ratio is usually the desired result.

This can be accomplished by a rapidly convergent iterative calculation if an additional measurement can be made of the noise background in the absence 


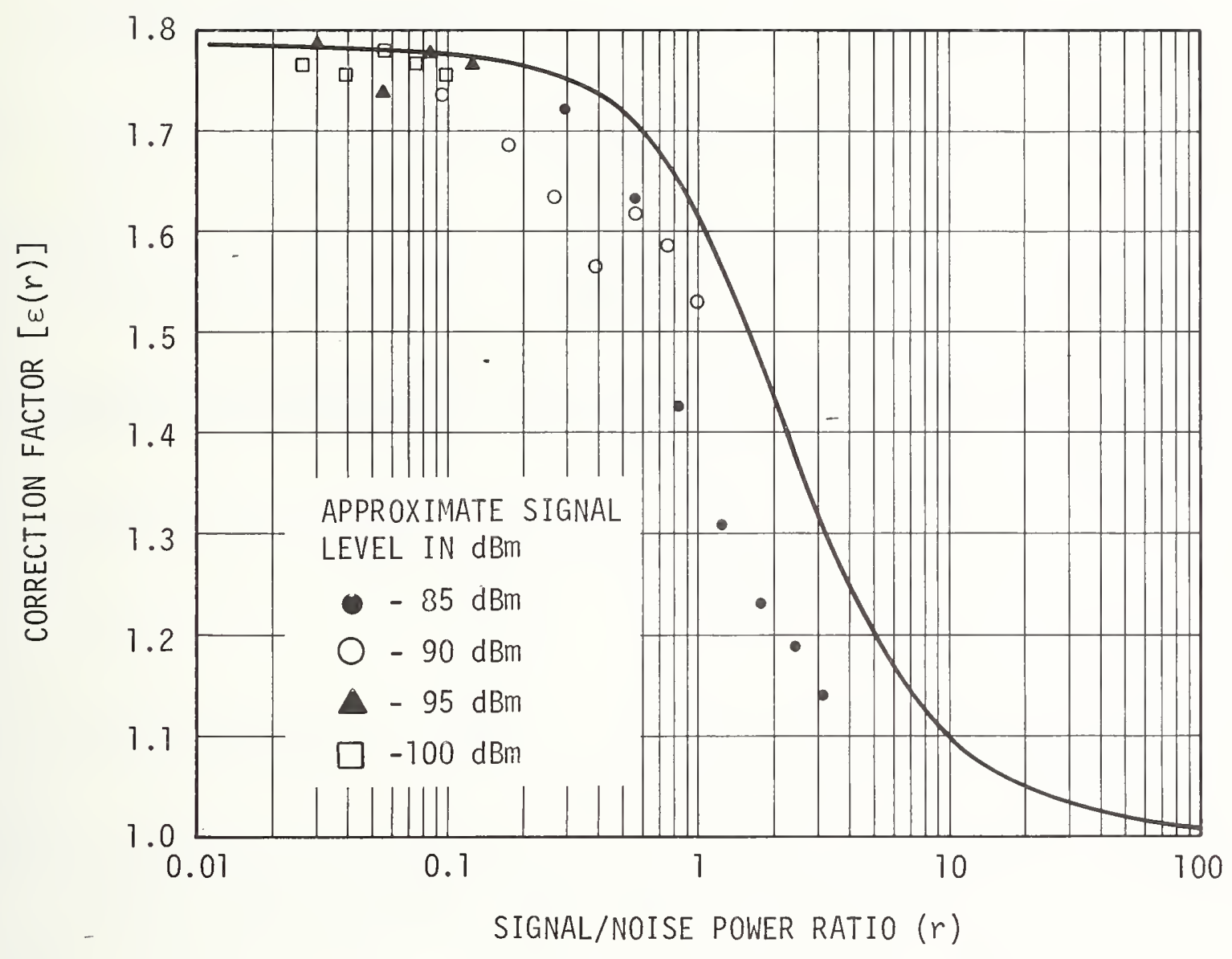

Fig. 1. Signāl/noise power ratio $(r)$ 
of signal. Consider the expression for the observed level of a signal consisting of signal power $S$ and noise power $N$. From eqs (28) and (34), we have

$$
\begin{aligned}
S+N & =P_{S+N} \varepsilon(S / N) \\
N & =P_{N} \varepsilon(0)
\end{aligned}
$$

where the P's are the relative observed levels. Subtracting and dividing, a transcendental relation in $S / N$ (or $r$ ) results.

$$
r=\frac{(S+N)-N}{N}=\frac{P_{S+N} \varepsilon(r)-P_{N} \varepsilon(0)}{P_{N} \varepsilon(0)}
$$

Because $\varepsilon(r)$ is a fairly slowly varying function of $r$, a simple iterative calculation will normally converge to $r$ within two or three iterations.

Other reference combinations of signal and noise can be used, but the one described above is probably the simplest and most widely useful. It is apparent that using the system noise level as a reference serves two additional purposes: First, it enables an absolute level calibration to be obtained if a standard noise source is available. Second, transference to the condition when $\mathrm{S}$ and $\mathrm{N}$ are of comparable level places the least stringent requirements upon system linearity.

\section{ACKNOWLEDGMENT AND NOTE}

The authors wish to acknowledge the helpful suggestion of $P . L$. Bender of the Joint Institute of Laboratory Astrophysics that enabled proceeding from eq (29a) to eq (29c). We are indebted to R. J. Matheson of the National Telecommunication and Information Agency's Institute for Telecommunication Sciences for the use of his Automatic Spectrum Analyzer and for his help in setting up and conducting the experiment. 


\section{REFERENCES}

[1] Dugundji, J., Envelopes and Pre-Envelopes of Real Waveforms, IRE Trans. PGIT IT-4, No. 1, 53 (March 1958).

[2] Erdelye, A., Magnus, W., Oberhettinger, F., and Tricomi, F. G., Tables of Integral Transforms, Vol. II, 252-253 (McGraw-Hill Book Co., Inc., New York, N.Y., 1954).

[3] Deutsch, R., Nonlinear Transformations of Random Processes, 10 (Prentice-Ha11, Inc., N.J., 1962).

[4] Lighthi11, M. J., Introduction to Fourier Analysis and Generalised Functions, 17 (Cambridge Press, England, 1962).

[5] Panter, P. F., Modulation, Noise, and Spectral Analysis, 114 (McGraw-Hill Book Co., Inc., New York, N.Y., 1965).

[6] Watson, G. N., Theory of Bessel Functions, 394 (Cambridge Press, England, 1962).

[7] Deutsch (1962) 7.

\subsection{General Reference}

Magrab, E. B. and Blomquist, D. S., The Measurement of Time-Varying Phenomena (Wiley-Interscience, N.Y., 1971). 
NBS-114A (REV. 9-78)

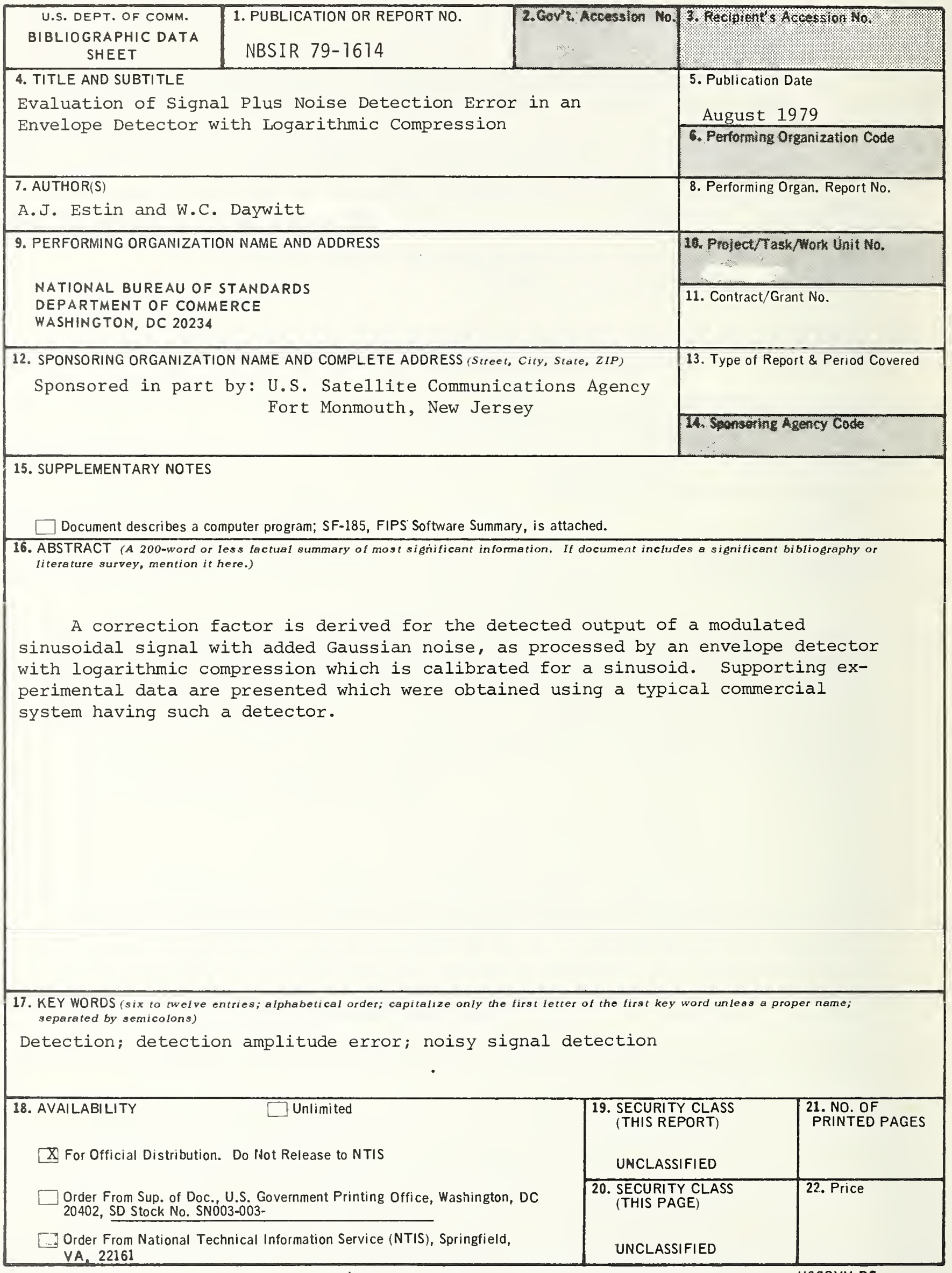



- 


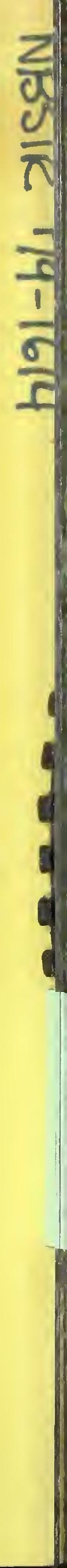

